\title{
Supporting Information: Tunable Plasmonic Neutral Density Filters and Chromatic Polarizers: Highly Packed 2D Arrays of Plasmonic Nanoparticle on Elastomer Substrate
}

\author{
Mahmoud A. Mahmoud* \\ Laser Dynamics Laboratory, School of Chemistry and Biochemistry, Georgia Institute of Technology, \\ Atlanta, Georgia 30332-0400 \\ *E-mail: $\underline{\text { mmahmoud@gatech.edu }}$
}

The seed-mediated technique was used to prepare the gold nanocubes. The seed solution was prepared by adding $600 \mu \mathrm{L}$ of an ice cold $0.01 \mathrm{M}$ sodium borohydride (sigma-Aldrich) solution to stirred $7.75 \mathrm{~mL}$ solution containing $0.1 \mathrm{M}$ cetyltrimethylammonium bromide (CTAB, SigmaAldrich) and $0.323 \mathrm{mM}$ hydrogen tetrachloroaurate trihydrate aqueous solution $\left(\mathrm{HAuCl}_{4} \cdot 3 \mathrm{H}_{2} \mathrm{O}\right.$, Sigma-Aldrich). In $1 \mathrm{~L}$ glass bottle, $\mathrm{HAuCl}_{4} \cdot 3 \mathrm{H}_{2} \mathrm{O}$ solution $(0.0394 \mathrm{~g}$ dissolved in $143 \mathrm{~mL} \mathrm{DI}$ water) was mixed CTAB solution (2.916 g dissolved in $400 \mathrm{~mL}$ DI water), and $7 \mathrm{~mL}(1 \mathrm{M})$ ascorbic acid (sigma-Aldrich). To the resulting solution, $0.35 \mathrm{~mL}$ of ten-fold diluted seed solution was added and allowed to grow for 4 hours.
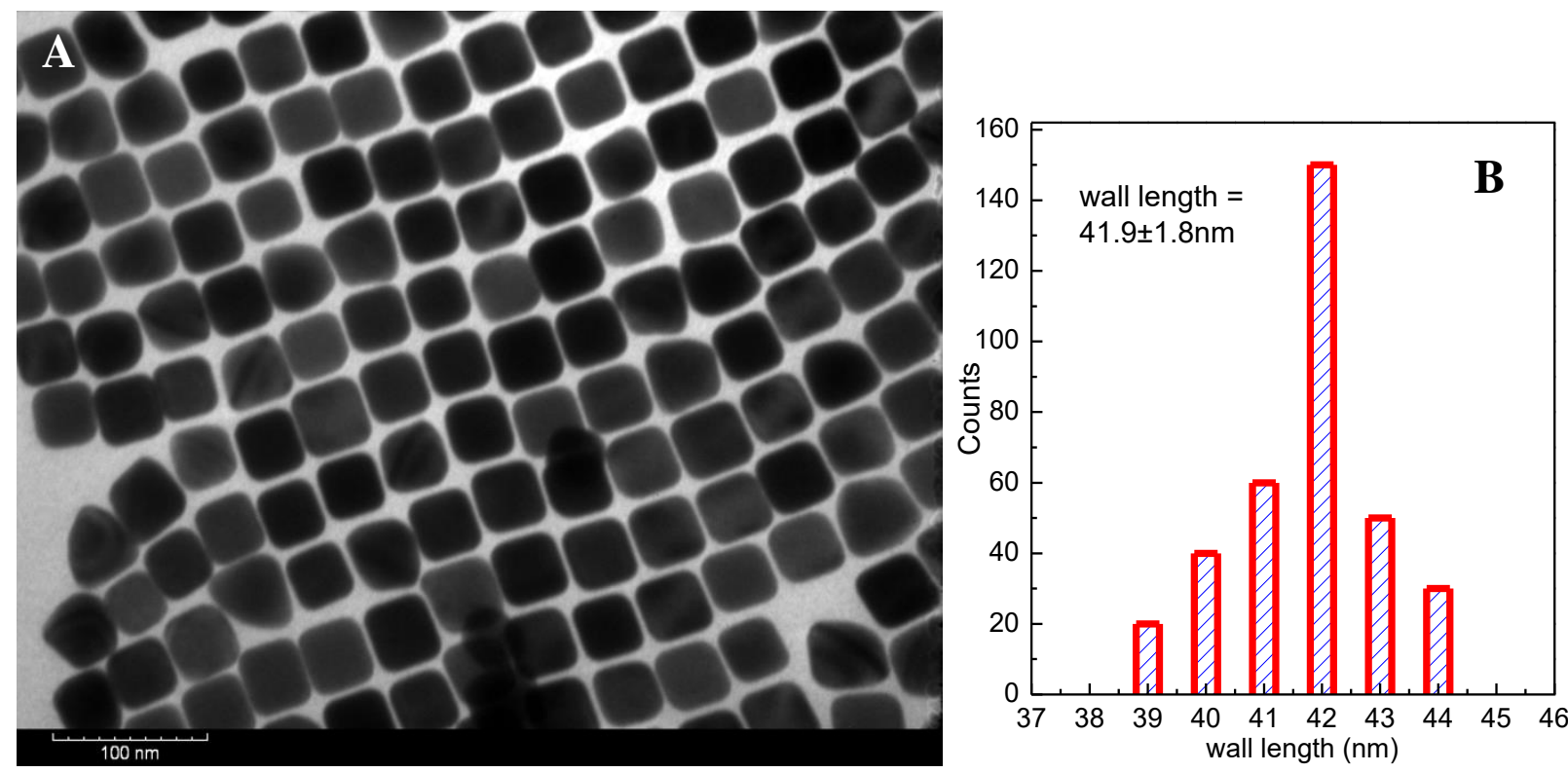

Figure S1A TEM image of the AuNCs after cleaning. B) Statistical analysis for the wall length of the AgNCs using imageJ software. The analysis carried out on 350 particles collected from 3 different TEM images showed that the wall length of the AuNC is $41.9 \pm 1.8 \mathrm{~nm}$. 


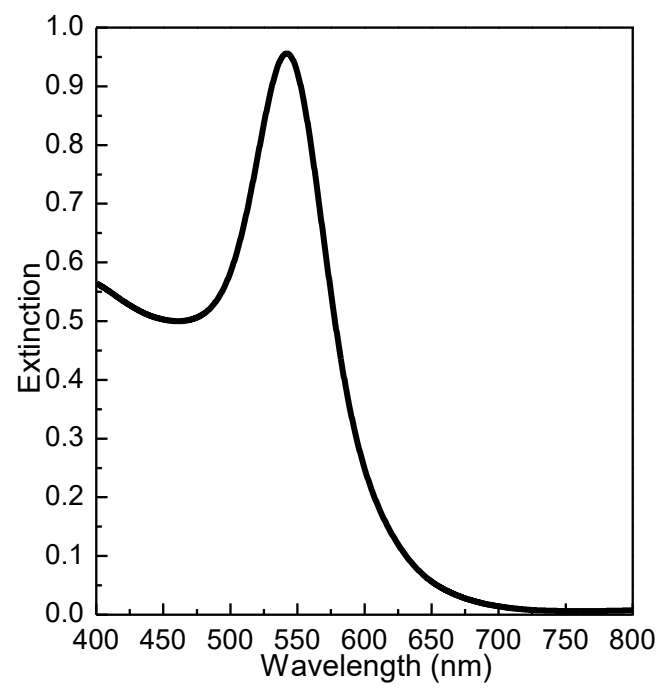

Figure S2 LSPR spectrum of gold nanocubes functionalized with PEG measured while dispersed in water.

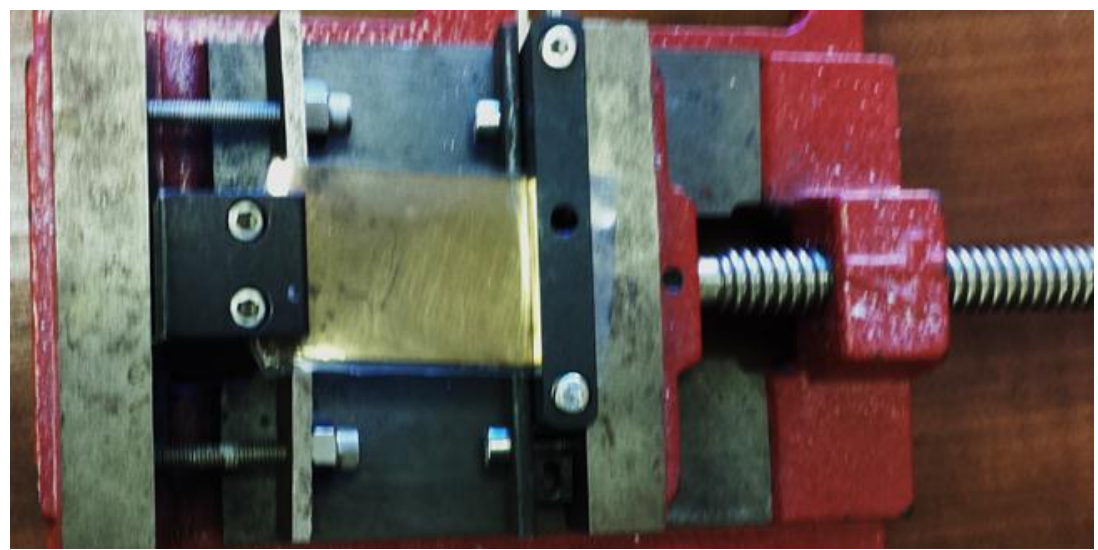

Figure S3 Homemade device used for stretching the arrays. 


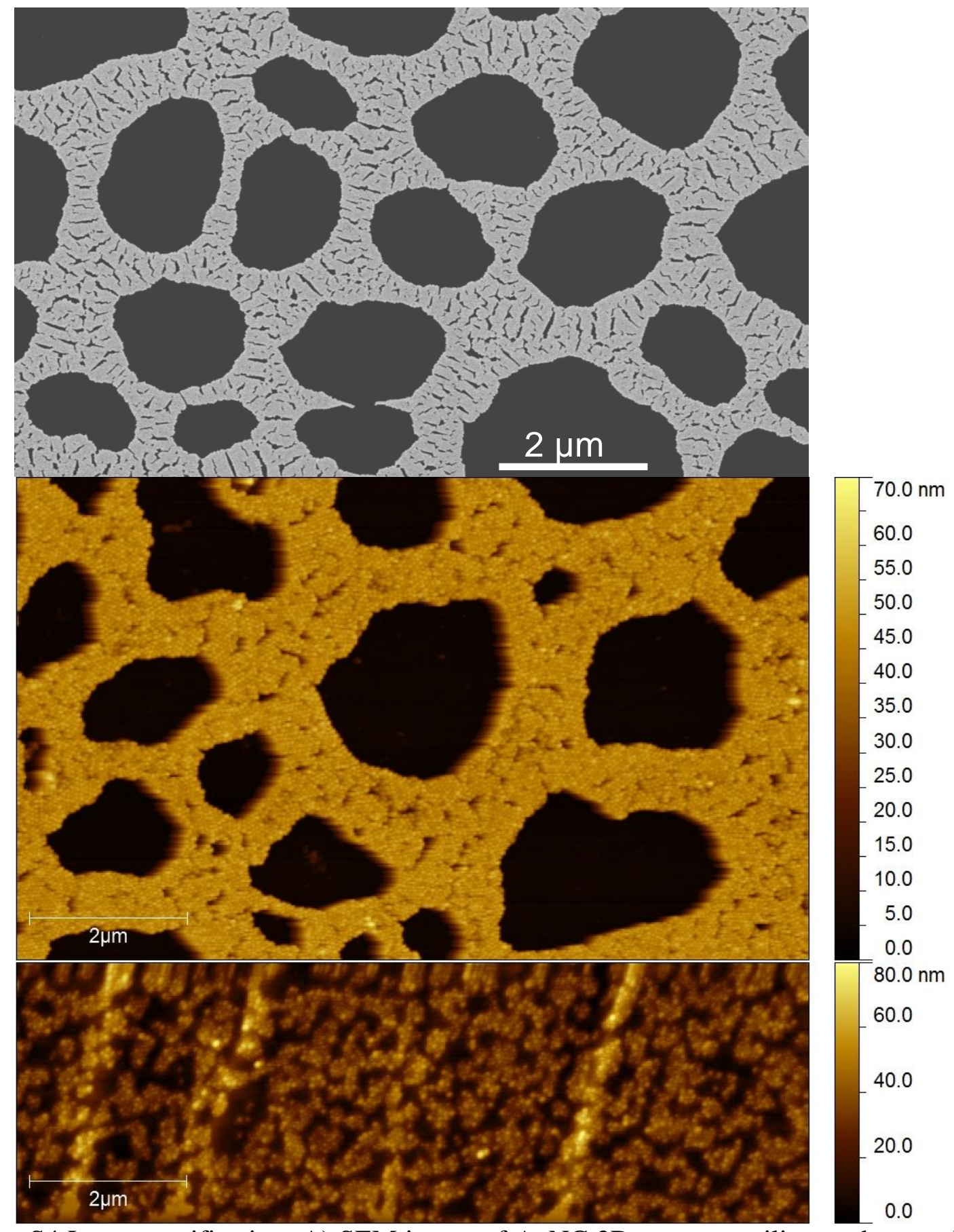

Figure S4 Low magnification: A) SEM image of AuNC 2D arrays on silicon substrate. B) AFM image of AuNC arrays on the surface of quartz coated with a thin film of PDMS. C) AFM image of AuNC arrays on the surface of PDMS substrate that was stretched 30 times. 


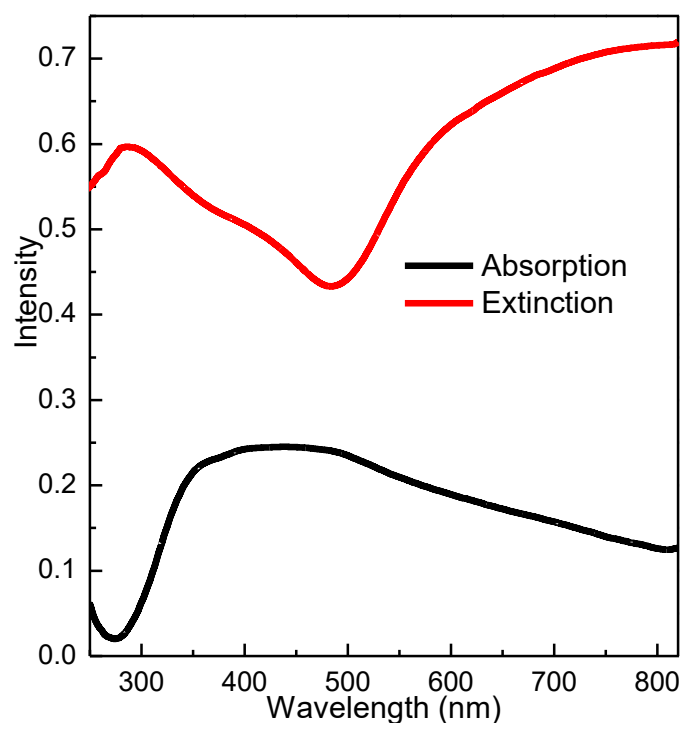

Figure S5 The absolute absorption spectrum and extinction spectrum of the AuNCs 2D arrays fabricated on the surface of quartz substrate.

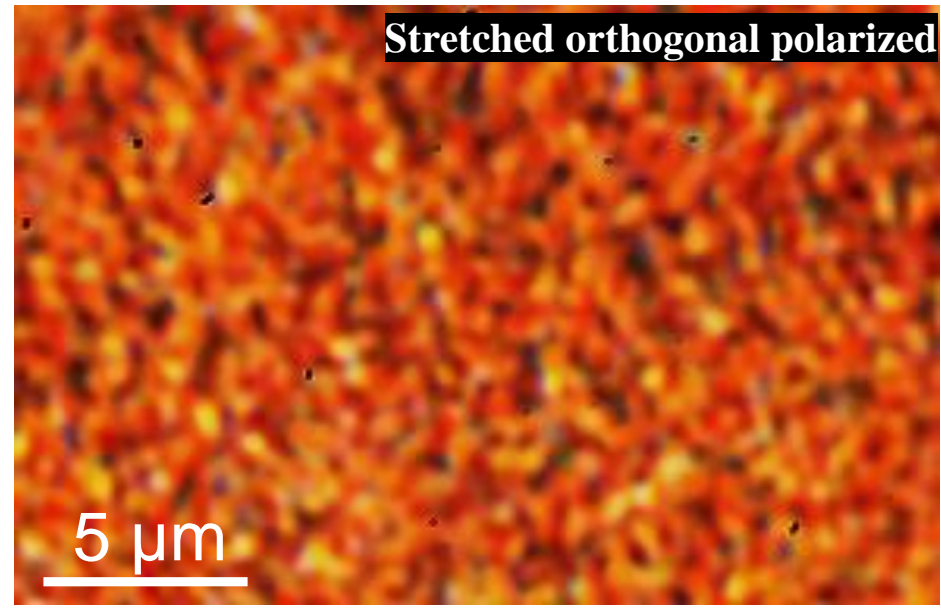

Figure S6 Dark field images of $45 \%$ stretched PNDF excited by orthogonally polarized light source. 